\title{
Targeting enrichment and correlation studies of glutathione and homocysteine in IgAVN patient urine based on a core-shell Zr-MOF
}

Xue, Ma ${ }^{\mathrm{a}}$, Jinghua Zhang ${ }^{\mathrm{b}}$, Chong Zhang ${ }^{\mathrm{a}}$, Xiaoqing Yang ${ }^{\mathrm{c}}$, Ajuan $\mathrm{Yu}^{\mathrm{a}}$, Yanjie Huang $^{c^{*}}$, Shusheng Zhang ${ }^{d}$, Gangfeng Ouyang ${ }^{d}$

a College of Chemistry, Key Laboratory of Molecular Sensing and Harmful Substances Detection Technology, Zhengzhou University, Kexue Avenue 100, Zhengzhou, Henan 450001, P. R. China.

${ }^{\mathrm{b}}$ College of medicine, Zhengzhou University, Kexue Avenue 100, Zhengzhou, Henan 450001, P. R. China.

${ }^{\mathrm{c}}$ Henan University of Traditional Chinese Medicine, Zhengzhou 4500 08, P. R. China.

${ }^{\mathrm{d}}$ Center of Advanced Analysis and Computational Science, Key Laboratory of Molecular Sensing and Harmful Substances Detection Technology, Zhengzhou University, Kexue Avenue 100, Zhengzhou, Henan 450001, P. R. China.

* Corresponding author.

E-mail address: yuajuan@zzu.edu.cn (Ajuan Yu), huangyanjie69@hotmail.com (Yanjie Huang).

* Corresponding author: Ajuan Yu

Tel.: +86-0371-67739608

Fax : +86-0371-6776224

E-mail : yuajuan@zzu.edu.cn 


\section{Index}

Table S1. Compound-dependent MS parameters for GSH and Hcy.

Table S2 Elemental analysis results of $\mathrm{SiO}_{2} @ 50$ Benz-Cys.

Figure S1. The energy dispersive X-ray spectroscopy (EDS) result of Si, O, and $\mathrm{Zr}$ elements of the $\mathrm{SiO}_{2} @ 50 \mathrm{Benz}-\mathrm{Cys}$.

Figure S2. XRD patterns of as-synthesized $\mathrm{SiO}_{2} @ 50 \mathrm{Benz}-\mathrm{Cys}, \mathrm{SiO}_{2} @ 50 \mathrm{Benz}, 50 \mathrm{Benz}-\mathrm{Cys}$ and $\mathrm{SiO}_{2}$ samples.

Figure S3. Thermogravimetric curve of $\mathrm{SiO}_{2} @ 50$ Benz-Cys, $\mathrm{SiO}_{2} @ 50 \mathrm{Benz}-\mathrm{HA}$.

Figure S4. Infrared spectra of the $\mathrm{SiO}_{2} @ 50 \mathrm{Benz}-\mathrm{Cys}$ after enrichment with sample at $\mathrm{pH}$ of 4-9.

Figure S5. SEM image of $\mathrm{SiO}_{2} @ 50 \mathrm{Benz}-\mathrm{Cys}$ after enrichment at $\mathrm{pH}$ of 4.

Table S3. Compared with other reported methods for aminothiols detection.

Table S4. One-Sample Kolmogorov-Smirnov Test.

Table S5. Test of Homogeneity of Variances.

HPLC-UV system and operating conditions

Reference 
Table S1. Compound-dependent MS parameters for GSH and Hcy.

\begin{tabular}{cccccc}
\hline Compound & MRM transition & DP (V) & EP (V) & CE (V) & CXP (V) \\
\hline \multirow{2}{*}{ GSH } & $308.1>179.1$ & 58 & 10 & 13 & 11 \\
& $308.1>233.1$ & 60 & 10 & 16 & 11 \\
\multirow{2}{*}{ Hcy } & $136.1>90.1$ & 46 & 10 & 10 & 11 \\
& $136.1>56.1$ & 53 & 10 & 12 & 11 \\
\hline
\end{tabular}

Table S2. Elemental analysis results of $\mathrm{SiO}_{2} @ 50 \mathrm{Benz}-\mathrm{Cys}$.

\begin{tabular}{ccccc}
\hline Sample & $\mathrm{N}(\mathrm{W} \%)$ & $\mathrm{C}(\mathrm{W} \%)$ & $\mathrm{H}(\mathrm{W} \%)$ & $\mathrm{S}(\mathrm{W} \%)$ \\
\hline $\mathrm{SiO}_{2} @ 50$ Benz-Cys & 1.24 & 4.75 & 1.76 & 0.96 \\
\hline
\end{tabular}

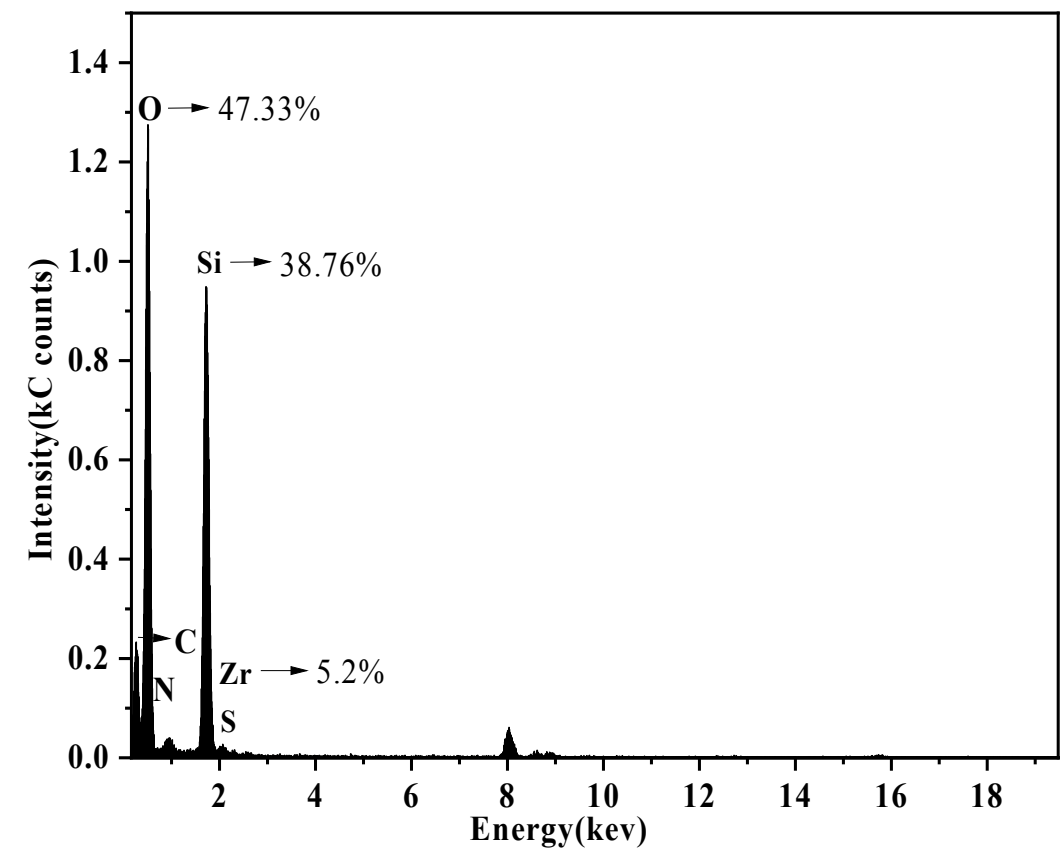

Figure S1. The energy dispersive X-ray spectroscopy (EDS) result of Si, O, and $\mathrm{Zr}$ elements of the $\mathrm{SiO}_{2} @ 50$ Benz-Cys. 


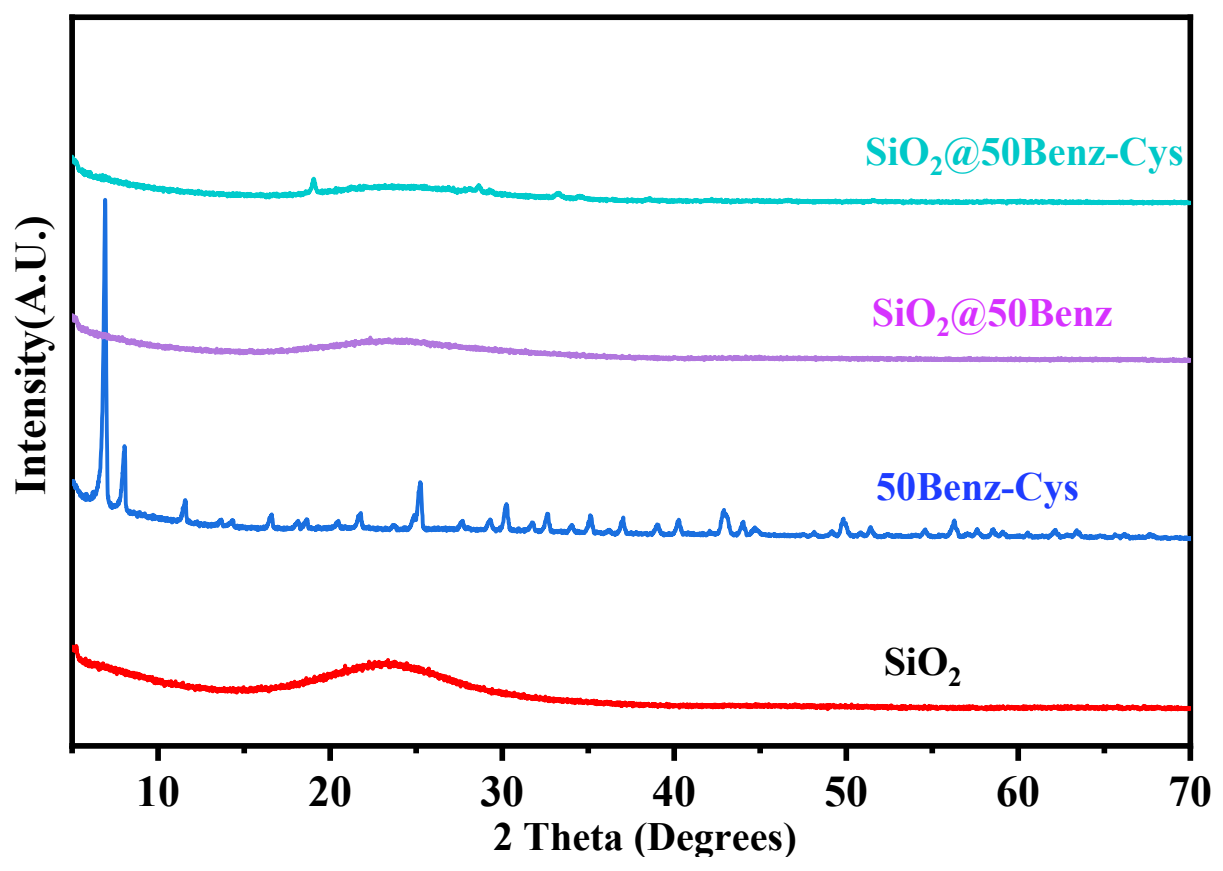

Figure S2.XRD patterns of as-synthesized $\mathrm{SiO}_{2} @ 50 \mathrm{Benz}-\mathrm{Cys}, \mathrm{SiO}_{2} @ 50 \mathrm{Benz}, 50 \mathrm{Benz}-\mathrm{Cys}$ and $\mathrm{SiO}_{2}$ samples.

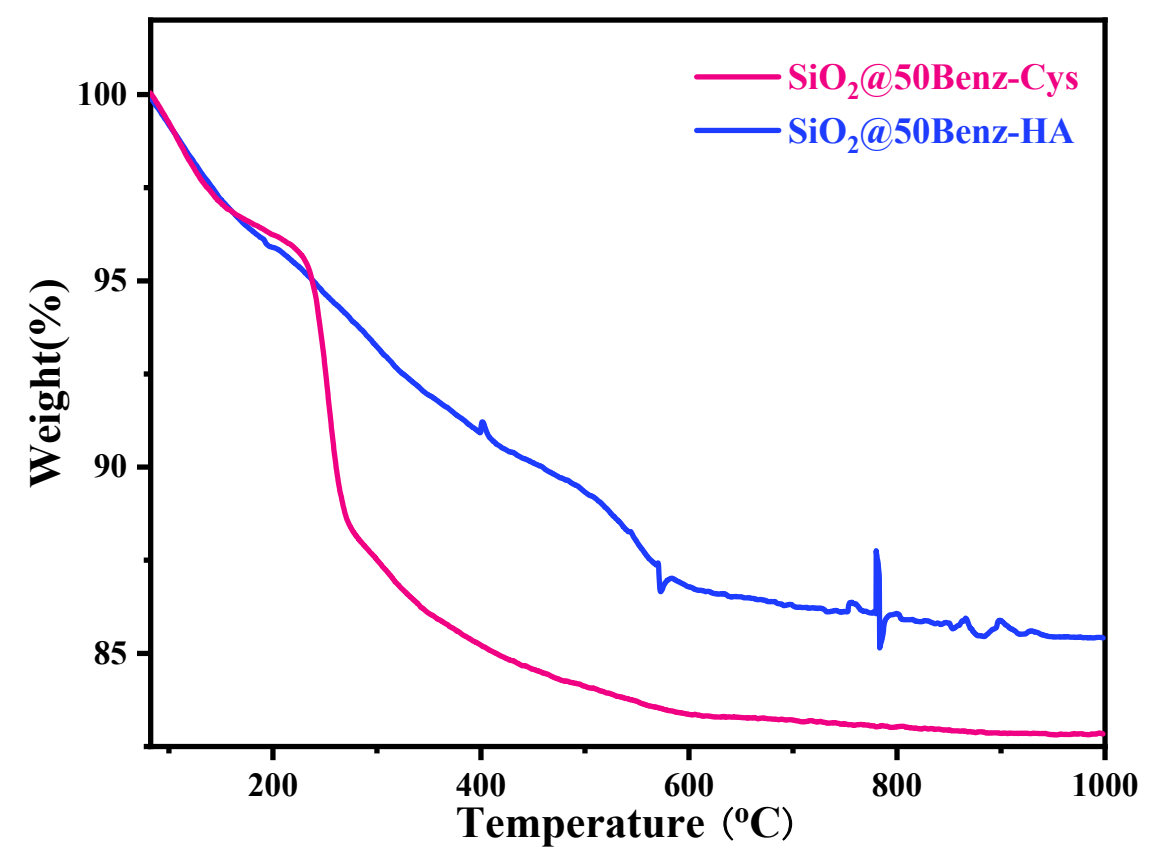

Figure S3. Thermogravimetric curve of $\mathrm{SiO}_{2} @ 50 \mathrm{Benz}-\mathrm{Cys}, \mathrm{SiO}_{2} @ 50 \mathrm{Benz}-\mathrm{HA}$. 


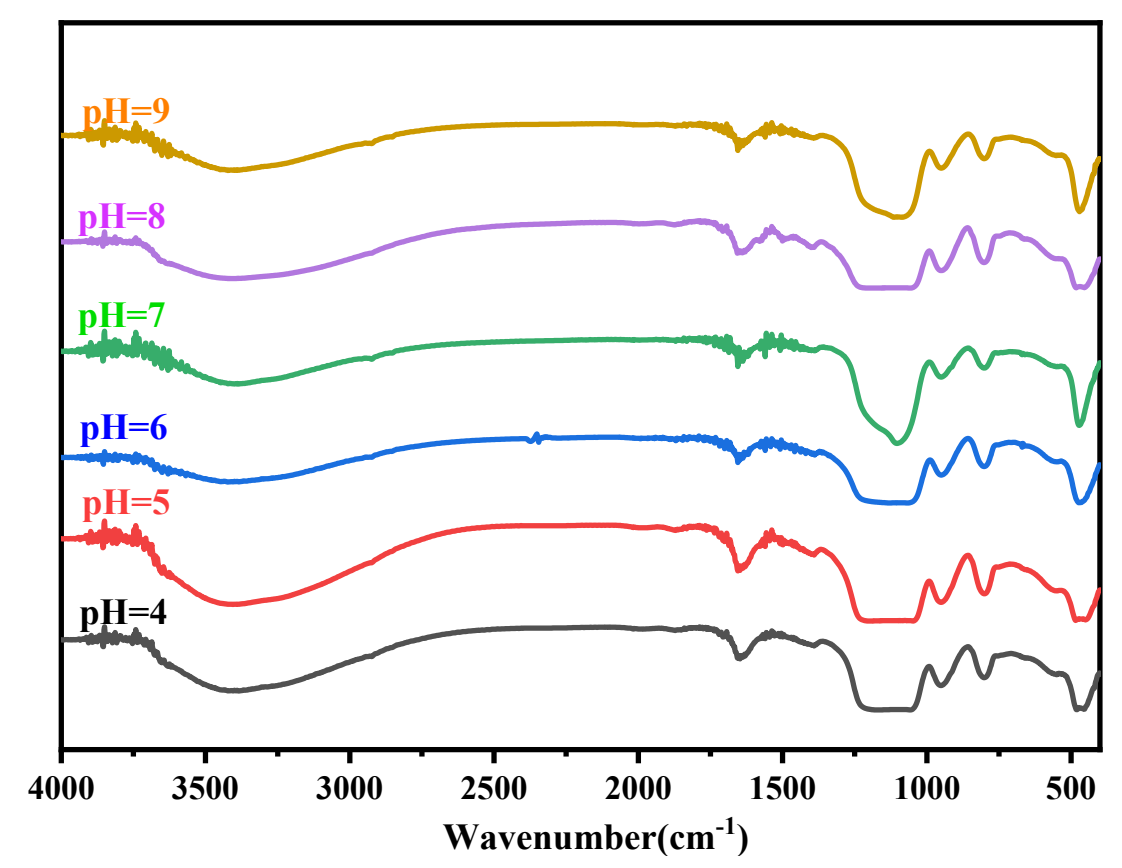

Figure S4. Infrared spectra of the $\mathrm{SiO}_{2} @ 50 \mathrm{Benz}-\mathrm{Cys}$ after enrichment with sample at pH of 4-9.

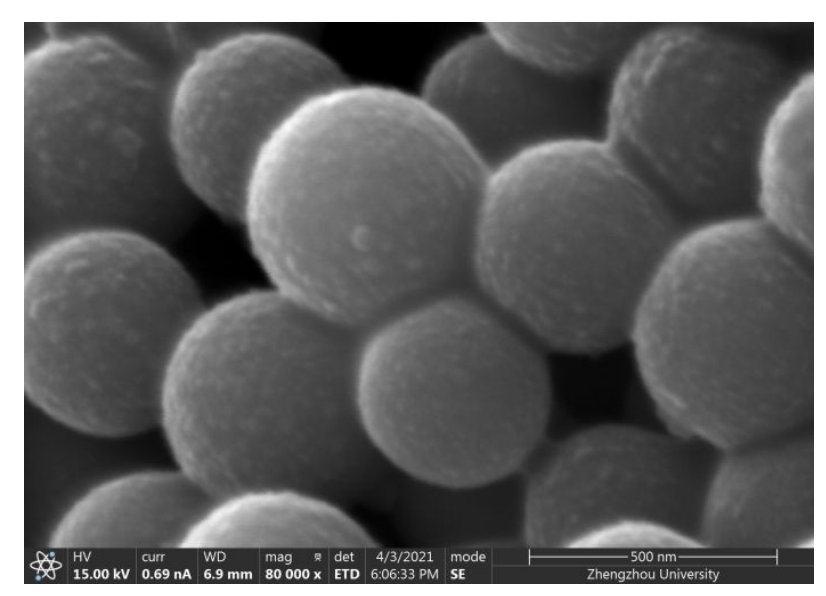

Figure S5. SEM image of $\mathrm{SiO}_{2} @ 50$ Benz-Cys after enrichment at pH of 4. 
Table S3. Compared with other reported methods for aminothiols detection.

\begin{tabular}{|c|c|c|c|c|c|c|c|}
\hline Material & Human Group & Sample & Analyte & Detection method & Recovery & LOD & Ref. \\
\hline AuNPs modified-monolith & healthy & $\begin{array}{l}\text { saliva } \\
\text { urine }\end{array}$ & GSH & Fluorescence & $86-105 \%$ & $5 \mathrm{nmol} \mathrm{L}^{-1}$ & [1] \\
\hline TCF-GSH & / & Hela cells & GSH & Fluorescence Probe & l & $0.45 \mu \mathrm{mol} \mathrm{L}^{-1}$ & {$[2]$} \\
\hline Tween 20-AuNPs & healthy & plasma & Aminothiols & $\begin{array}{c}\text { capillary } \\
\text { electrophoresis }\end{array}$ & $97.0-99.5 \%$ & $12 \mathrm{nmol} \mathrm{L}^{-1}$ & {$[3]$} \\
\hline $2 \times$ Tween 20 -AuNPs & healthy & urine & Aminothiols & CE-LIF & $96.8-102.8 \%$ & $4 \mathrm{nmol} \mathrm{L}^{-1}$ & {$[4]$} \\
\hline $\begin{array}{l}\text { Micro Reduced Glutathione } \\
\text { (GSH) Assay Kit }\end{array}$ & l & Cells & GSH & Colorimetric Method & l & $3.763 \mu \mathrm{g} \mathrm{mL}^{-1}$ & {$[5]$} \\
\hline GSH Assay Kit & / & crab hepatopan-creatic & GSH & Colorimetric Method & / & $0.5 \mu \mathrm{mol} \mathrm{L}^{-1}$ & {$[6]$} \\
\hline $\begin{array}{l}\text { GSH (Glutathione) ELISA } \\
\text { Kit }\end{array}$ & / & Cells & GSH & ELISA & l & $0.94 \mu \mathrm{g} \mathrm{mL}^{-1}$ & {$[7]$} \\
\hline Glutathione Assay Kit & / & Serum & GSH & Fluorescent & / & l & {$[8]$} \\
\hline homocysteine ELISA kit & / & plasma & Hcy & Colorimetric & l & $10 \mathrm{ng} \mathrm{mL}^{-1}$ & [9] \\
\hline $\mathrm{SiO}_{2} @ 50 \mathrm{Benz}-\mathrm{Cys}$ & $\begin{array}{l}\text { Healthy } \\
\text { Patient }\end{array}$ & Urine & $\begin{array}{l}\text { GSH } \\
\text { Hcy }\end{array}$ & HPLC-MS/MS & $79.5-105 \%$ & $\begin{array}{c}0.5 \mathrm{nmol} \mathrm{L}^{-1} \\
1 \mathrm{nmol} \mathrm{L}^{-1}\end{array}$ & $\begin{array}{l}\text { This } \\
\text { work }\end{array}$ \\
\hline
\end{tabular}


Table S4. One-Sample Kolmogorov-Smirnov Test

\begin{tabular}{|c|c|c|c|c|c|c|c|}
\hline & & $\begin{array}{c}\text { GSH } \\
\text { in Control }\end{array}$ & $\begin{array}{c}\text { Hcy } \\
\text { in Control }\end{array}$ & $\begin{array}{c}\text { GSH } \\
\text { in IgAV }\end{array}$ & $\begin{array}{c}\text { Hcy } \\
\text { in IgAV }\end{array}$ & $\begin{array}{c}\text { GSH } \\
\text { in IgAVN }\end{array}$ & $\begin{array}{c}\text { Hcy } \\
\text { in IgAVN }\end{array}$ \\
\hline \multicolumn{2}{|c|}{$\mathrm{N}$} & 17 & 17 & 11 & 11 & 12 & 12 \\
\hline \multirow[b]{2}{*}{$\begin{array}{c}\text { Normal } \\
\text { Parameters }{ }^{\mathrm{a}, \mathrm{b}}\end{array}$} & Mean & 6.9694 & 10.1812 & 1.8855 & 26.5982 & 2.6508 & 34.885 \\
\hline & $\begin{array}{c}\text { Std. } \\
\text { Deviation }\end{array}$ & 0.2725 & 0.41088 & 0.16759 & 0.63534 & 0.32197 & 0.25461 \\
\hline Most & Absolute & 0.236 & 0.23 & 0.147 & 0.303 & 0.332 & 0.206 \\
\hline Extreme & Positive & 0.231 & 0.147 & 0.122 & 0.234 & 0.332 & 0.188 \\
\hline Differences & Negative & -0.236 & -0.23 & -0.147 & -0.303 & -0.306 & -0.206 \\
\hline \multicolumn{2}{|l|}{ Test Statistic } & 0.236 & 0.23 & 0.147 & 0.303 & 0.332 & 0.206 \\
\hline \multicolumn{2}{|c|}{ Asymp. Sig. (2-tailed) } & $0.013^{\mathrm{c}}$ & $0.017^{\mathrm{c}}$ & $0.200^{\mathrm{c}, \mathrm{d}}$ & $0.006^{\mathrm{c}}$ & $0.001^{\mathrm{c}}$ & $0.169^{\mathrm{c}}$ \\
\hline
\end{tabular}

a. Test distribution is Normal.

b. Calculated from data.

c. Lilliefors Significance Correction.

d. This is a lower bound of the true significance.

Abbreviations: GSH, glutathione; Hcy, homocysteine; IgAV, immunoglobulin A vasculitis; IgAVN, immunoglobulin A vasculitis with nephritis.

Table S5. Test of Homogeneity of Variances

\begin{tabular}{ccccc}
\hline & $\begin{array}{c}\text { Levene } \\
\text { Statistic }\end{array}$ & df1 & df2 & Sig. \\
\hline GSH & 2.085 & 2 & 37 & 0.139 \\
Hcy & 0.206 & 2 & 37 & 0.815 \\
\hline
\end{tabular}

Abbreviations: GSH, glutathione; Hcy, homocysteine.

\section{HPLC-UV system and operating conditions}

The HPLC system was a Shimadzu LC-20A with UV detection. Chromatographic separation was performed with Agilent 5TC-C18(2) $(250 \times 4.6 \mathrm{~mm})$ at $40{ }^{\circ} \mathrm{C}$. The 
detection wavelength was $280 \mathrm{~nm}$. The mobile phase consisted of water (A) and acetonitrile (B) and was previously filtered through a Millipore filtration system. The mobile phase, delivered at a flow rate of $1.0 \mathrm{~mL} \mathrm{~min}{ }^{-1}$, was programmed as follows: 0 $\min , 1 \% \mathrm{~B} ; 5 \min , 1 \% \mathrm{~B} ; 7 \mathrm{~min}, 5 \% \mathrm{~B} ; 17 \mathrm{~min}, 40 \% \mathrm{~B} ; 27 \mathrm{~min}, 95 \% \mathrm{~B} ; 32 \mathrm{~min}, 95 \%$ $\mathrm{B} ; 33 \mathrm{~min}, 5 \% \mathrm{~B} ; 35 \mathrm{~min}, 5 \%$. Equilibration time was $5 \mathrm{~min}$ prior to the next injection. The total run time was $35 \mathrm{~min}$.

\section{Reference}

[1] Mompo-Rosello O.; Vergara-Barberan M.; Simo-Alfonso E. F.; Herrero-Martinez J. M. In syringe hybrid monoliths modified with gold nanoparticles for selective extraction of glutathione in biological fluids prior to its determination by HPLC. Talanta, 2020, 209, 120566.

[2] Sedgwick A. C.; Gardiner J. E.; Kim, G.; Yevglevskis M.; Lloyd M. D.; Jenkins A. T. A.; Bull S. D.; Yoon J.; James T. D. Long-wavelength TCF-based fluorescence probes for the detection and intracellular imaging of biological thiols. Chem Commun (Camb) 2018, 54, 4786-4789.

[3] Chang, C.-W.; Tseng, W.-L. Gold Nanoparticle Extraction Followed by Capillary Electrophoresis to Determine the Total, Free, and Protein-Bound Aminothiols in Plasma. Anal. Chem. 2010, 82, 2696-2702.

[4] Shen C.-C.; Tseng W.-L.; Hsieh M.-M. Selective enrichment of aminothiols using polysorbate 20-capped gold nanoparticles followed by capillary electrophoresis with laser-induced fluorescence, $J$ Chromatogr A 2009, 1216, 288-293.

[5] Gongsun, X.; Zhao, Y.; Jiang, B.; Xin, Z.; Shi, M.; Song, L.; Qin, Q.; Wang, Q.; Liu, X.; Inhibition of MUC1-C regulates metabolism by AKT pathway in esophageal squamous cell carcinoma. J. Cell. Physiol. 2019, 234, 12019-12028.

[6] Wang, L.; Yan, B.; Liu, N.; Li, Y.; Wang, Q. Effects of cadmium on glutathione synthesis in hepatopancreas of freshwater crab, Sinopotamon yangtsekiense. Chemosphere 2008, 74, 51-56.

[7] Yang J.; Yang Y.; Tian, L.; Sheng, X.-F.; Liu, F.; Cao, J.-G. Casticin-induced apoptosis involves death receptor 5 upregulation in hepatocellular carcinoma cells. World J Gastroentero. 2011, 17, 4298-4307.

[8] Manfredini, F.; Straudi, S.; Lamberti, N.; Patergnani, S.; Tisato, V.; Secchiero, P.; Bernardi, F.; Ziliotto, N.; Marchetti, G.; Basaglia, N.; Bonora, M.; Pinton, P. Rehabilitation Improves Mitochondrial Energetics in Progressive Multiple Sclerosis: The Significant Role of Robot-Assisted Gait Training and of the Personalized Intensity. Diagnostics 2020, 10, 834.

[9] Toriumi, K.; Berto, S.; Koike, S.; Usui, N.; Dan, T.; Suzuki, K.; Miyashita, M.; Horiuchi, Y.; Yoshikawa, A.; Asakura, M.; Nagahama, K.; Lin, H.-C.; Sugaya, Y.; Watanabe, T.; Kano, M.; Ogasawara, Y.; Miyata, T.; Itokawa, M.; Konopka, G.; Arai, M. Combined glyoxalase 1 dysfunction and vitamin B6 deficiency in a schizophrenia model system causes mitochondrial dysfunction in the prefrontal cortex. Redox Biol. 2021, 45, 102057. 